\title{
Book review: Exploring thinking as communicating in CSCL
}

\author{
Anna Sfard, Thinking as communicating: human development, \\ the growth of discourses and mathematizing, Cambridge University \\ Press, 2008
}

\section{Gerry Stahl}

Published online: 12 August 2008

(C) International Society of the Learning Sciences, Inc.; Springer Science + Business Media, LLC 2008

Anna Sfard raised the methodological discourse in the CSCL community to a higher niveau of self-understanding a decade ago with her analysis of our two prevalent metaphors for learning: the acquisition metaphor (AM) and the participation metaphor (PM). Despite her persuasive argument in favor of PM and a claim that AM and PM are as incommensurable as day and night, she asked us to retain the use of both metaphors and to take them as complementary in the sense of the quantum particle/wave theory, concluding that

Our work is bound to produce a patchwork of metaphors rather than a unified, homogenous theory of learning. (Sfard 1998, p. 12)

A first impression of her new book is that she has herself now come closer than one could have then imagined to a unified, homogenous theory of learning. It is a truly impressive accomplishment, all the more surprising in its systematic unity and comprehensive claims given her earlier discussion. Of course, Sfard does not claim to give the last word on learning, since she explicitly describes how both learning and theorizing are in principle open-ended. One could never acquire exhaustive knowledge of a domain like math education or participate in a community culture in an ultimate way, since knowledge and culture are autopoietic processes that keep building on themselves endlessly.

Sfard does not explicitly address the tension between her earlier essay and her new book. To reconcile her two discourses and to assess their implications for the field of CSCL, one has to first review her innovative and complex analysis of mathematical thinking.

\section{Understanding math objects}

Sfard introduces her presentation by describing five quandaries of mathematical thinking. I will focus on just one of these, which seems particularly foundational for a theory of math cognition, although all are important for math education: What does it mean to understand

G. Stahl $(\bowtie)$

Drexel University, Philadelphia, PA, USA

e-mail: G.Stahl@drexel.edu 
something in mathematics? Sometimes we ask, What is deep understanding in math (as opposed to just being able to go through the procedures)? I am particularly interested in this question because in my research group we are observing the chat of an algebra student who repeatedly says things like, "the formula makes sense to me...but I do not see why it should either" (see chat screenshot in Fig. 1). For us as analysts, it is hard to know how Aznx cannot see why the equation is right if it makes sense to him; the nature of his understanding seems to be problematic for him as well as for us. One assumes that either he "possesses" knowledge about the applicability of the formula or he does not.

According to Sfard's theory, a math object-like the equation that Bwang is proposing in the chat for the number of blocks in stage $\mathrm{N}$ of a specific kind of pyramid-is an objectification or reification of a discursive process, such as counting the blocks at each stage (see also Wittgenstein 1944/1956, p.3f, §3). In fact, we observe the team of students in the chat environment visibly constructing the pyramid in their shared whiteboard. Looking through Sfard's eyes, we can watch the students counting in a variety of ways, sometimes by numbering the graphical representations of blocks, other times by referencing shared drawings of the blocks from the chat postings, or by coordinating the sequential drawing of arranged blocks with the chat discussion in ways that make visible to the other students the enumeration of the pattern.

Sfard's central chapters spell out the ways in which math objects are subsequently coconstructed from these counting communication processes, using general procedures she names saming, reification and encapsulation. Note, for instance, that Bwang is explicitly engaged in a process of saming: claiming that a set of already reified math objects (previous

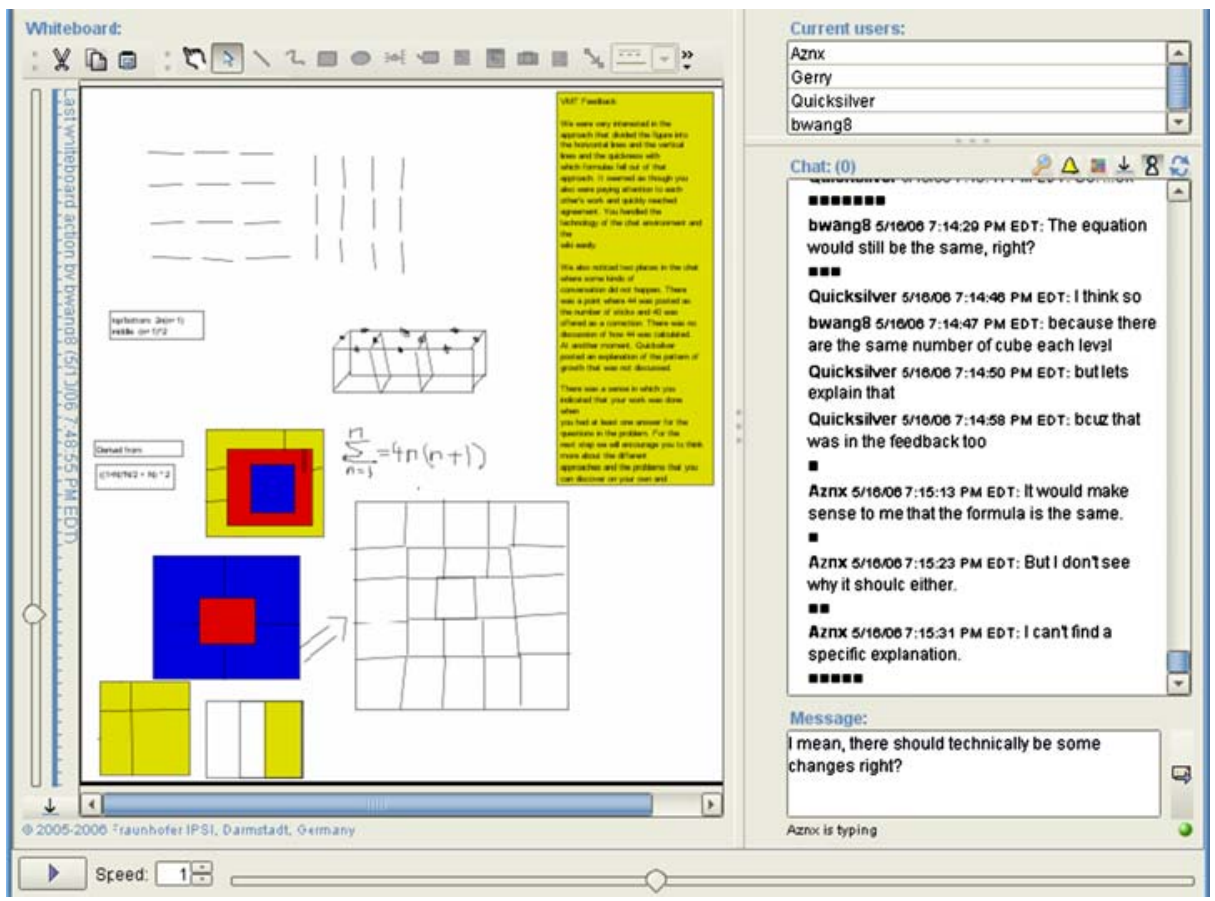

Fig. 1 Three students chat about the mathematics of various formations of stacked blocks. Aznx expresses uncertainty about his understanding of Bwang's proposal about a formula and his ability to explain the formula in response to Quicksilver 
and current equations the students are discussing) are "the same." He states, "The equation would still be the same, right?... Because there are the same number of cube[s on] each level." He has reified the counting of the blocks into the form of a symbolic algebraic expression, which looks like an object with investigable attributes, rather than a discursive counting process. If he were a more expert speaker of math discourse, Bwang might even encapsulate the whole set of same equations as a new object, perhaps calling them pyramid equations. And so it goes.

In our case study, Aznx, Bwang and Quicksilver engage in four hours of online collaborative math discourse. They consider patterns of several configurations of blocks that grow step by step according to a rule (see also Moss and Beatty 2006). They develop recursive and quadratic expressions for the count of blocks and number of unduplicated sides in the patterns. They decide what to explore and how to go about it, and they check and question each other's math proposals, collaboratively building shared knowledge. Their group knowledge ${ }^{1}$ is fragile, and the team repeatedly struggles to articulate what they have found out and how they arrived at it, encouraged to explain their work by the facilitator, who placed the textbox of feedback in their whiteboard. During their prolonged interaction, the group creates a substantial set of shared drawings and chat postings, intricately woven together in a complex web of meaning.

Sfard describes the discursive construction of math objects, which — as Husserl (1936/ 1989) said-is sedimented in the semiotic objects themselves. To paraphrase and reify Sfard's favorite Wittgenstein quote, ${ }^{2}$ the use (the construction process) is embodied in the sign as its meaning. She lays out the generative process by which a tree of realizations is built up through history and then reified by a new symbolic realization that names the tree. The algebraic equation that Bwang proposes is one such symbolic expression. The students have built it to encapsulate and embody various counting processes and graphical constructions that they have produced together. The equation also incorporates earlier math objects that the group has either co-constructed or brought into their discourse from previous experience (e.g., Gauss' formula for the sum of $N$ consecutive integers, previously learned in their math classrooms).

A centerpiece of Sfard's theory is the definition of a math object as the recursive tree of its manifold visual realizations. I will not attempt to summarize her argument because I want to encourage you to read it first hand. It is presented with all the grace, simplicity, insight and rigor of an elegant mathematical proof. It is itself built up from quasi-axiomatic principles, through intermediate theorems, illustrated with persuasive minimalist examples.

It is this definition of math object that, I believe, provides the germ of an answer to the conundrum of deep math understanding. That is, to understand a math object is to understand the realizations of that object. One must be able to unpack or de-construct the processes that are reified as the object. To be able to write an equation-e.g., during a test in school, where the particular equation is indicated-is not enough. One must to some extent be able to re-create or derive the equation from a concrete situation and to display

\footnotetext{
${ }^{1}$ The use of the term group cognition for referring to the discursive methods that small groups collaboratively use to accomplish cognitive tasks like solving problems often raises misunderstandings because readers apply AM when they see the noun cognition. They wonder where the acquired cognitive objects are possessed and stored, since there is no individual physical persisting agent involved. If one applies PM instead, in line with Sfard's theory, then it makes much more sense that discursive objects are being built up within a publicly available group discourse.

2 "For a large class of cases - though not for all — in which we employ the word 'meaning' it can be defined thus: the meaning of a word is its use in the language." (1953, p. 20, §43)
} 
alternative visual realizations, such as graphs, formulas, special cases and tables of the equation. There is not a single definition of the equation's meaning, but a network of interrelated realizations. To deeply understand the object, one must be conversant with multiple such realizations, be competent at working with them, be cognizant of their interrelationships and be able to recognize when they are applicable.

\section{Routines of math discourse}

Sfard then moves from ontology to pedagogy - from theory of math objects to theory of discourses about such objects, including how children come to participate in these discourses and individualize the social language into their personal math thinking. Based on her intensive work with data of young children learning math, she describes with sensitivity and insight how children come to understand words like number, same, larger and other foundational concepts of mathematical cognition. It is not primarily through a rationalist process of individual, logical, mental steps. It is a discursive social process: not acquisition of knowledge, but participation in co-construction of realizations. Sfard describes this as participation in social routines - much like Wittgensteinian language games. She describes in some detail three types of routines: deeds, explorations and rituals. Routines are metalevel rules that describe recurrent patterns of math discourse. Like Sfard's discussion itself, they describe math discourses rather than math objects. Deeds are methods for making changes to objects, such as drawing and enumerating squares on the whiteboard. Explorations are routines that contribute to a theory, like Bwang's proposal.

Rituals, by contrast are socially oriented. The more we try to understand Aznx's chat postings, the more we see how engaged he is in social activity rituals. He provides group leadership in keeping the group interaction and discourse moving, reflecting, explaining, responding to the facilitator, positioning his teammates, assigning tasks to others. His mathematical utterances are always subtly phrased to maintain desirable social relations within the group and with the facilitator-saving face, supporting before criticizing, leaving ignorances ambiguous, checking in with others on their opinions and understandings, positioning his teammates in the group interaction and assigning tasks to others. Each utterance is simultaneously mathematical and social, so that one could not code it (except for very specific purposes) as simply content, social or off-topic once one begins to understand the over-determined mix of work it is doing in the discourse. Similarly, Bwang's explicitly mathematical proposals (explorations) are always intricately situated in the social interactions. Quicksilver often reflects on the group process, articulating the group routines to guide the process. Sfard's analysis helps us see the various emergent roles the students' participations play in their discourse - without requiring us to reduce the complexity of the social and semantic interrelationships.

Just as Vygotsky $(1930 / 1978,1934 / 1986)$ noticed that children start to use new adult words before they fully understand the meaning of the words (in fact, they learn the meaning by using the word), so Sfard argues that children advance from passive use of math concepts to routine-driven, phrase-driven and finally object-driven use. They often begin to individualize group knowledge and terminology through imitation. Again, the part of the book on routines requires and deserves careful study and cannot be adequately presented in a brief review. I would encourage trying to apply Sfard's analysis to actual data of children learning math.

In our case, we see Aznx imitating his partners' routines and thereby gradually individualizing them as his own abilities. He often makes a knowledgeable-sounding 
proposal and then questions his own understanding. He does not possess the knowledge, but he is learning to participate in the discourse. In a collaborative setting, his partners can correct or accept his trials, steering and reinforcing his mimetic learning. During our fourhour recording, we can watch the group move through different stages of interaction with the symbols and realizations of math objects. The students we observe are not fully competent speakers of the language of math; as they struggle to make visible to each other (and eventually through that to themselves) their growing understanding, we as analysts can see both individual understanding and group cognition flowering. We can make sense of the discourse routines and interactional methods with the help of Sfard's concepts.

Participation in the discourse forms of math routines - such as exploration, ritual and imitation - can expose students to first-hand experiences of mathematical meaning making and problem solving. As they individualize these social experiences into their personal discourse repertoire, they thereby construct the kind of deep understanding that is often missing from acquisitionist/transmission math pedagogies (see Lockhart 2008, for a critique of the consequences of AM schooling).

\section{Situating math discourse}

Sfard's theory resolves many quandaries that have bothered people about participationist and group cognitive theories. How can ideas exist in discourses and social groupings rather than in individual minds? It provides detailed analyses of how people participate in the discourses of communities - at least within the domain of math discourses, both local and historical. It provides an account of some basic ways in which individual learning arises from collaborative activities. It indicates how meaning (as situated linguistic use) can be encapsulated in symbols. It explains how children learn, and that creativity is possible, while suggesting ways to foster and to study learning. It describes some of the mediations by which public discourses - as the foundational form of knowledge and group cognitionevolve and are individuated into private thinking.

Sfard has done us the great service of bringing the "linguistic turn" of twentieth century philosophy (notably Wittgenstein) into twenty-first century learning science, elaborating its perspective on the challenging example of math ed. She shows how to see math concepts and student learning as discourse phenomena rather than mental objects.

The kind of theoretical undertaking reported in this book must restrict its scope in order to tell its story. However, if we want to incorporate its important accomplishments into CSCL research, then we must also recognize its limitations and evaluate its contributions vis a vis competing theories. In addition to noting its incomplete treatment of sociocognitive theory, knowledge building, activity theory, ethnomethodology or distributed cognition, for instance, we should relate it more explicitly to the characteristics of CSCL.

First CSCL. By definition of its name, CSCL differs from broader fields of learning in two ways: its focus on collaborative learning (e.g., small group peer learning) and its concern with computer support (e.g., asynchronous online discussion, synchronous text chat, wikis, blogs, scripted environments, simulations, mobile computing, video games). Sfard does not present examples of small group interaction; her brief excerpts are from dyadic face-to-face discussions or adult-child interviews. Her empirical analyses zero in on individual math skills and development, rather than on the group mechanisms by which contributions from different personal perspectives are woven together in shared discourse. We now need to extend her general approach to computer-mediated interaction within small groups of students working together on the construction and deconstruction of math objects. 
Fine-grained analysis of collaboration requires high-fidelity recordings, which - as Sfard notes - must be available for detailed and repeated study. She makes the tantalizing hypothesis that Piaget's famous distinction between successive developmental stages in children's thinking during his conservation experiments may be a misunderstanding caused by his inability to re-view children's interactions in adequate detail. Tape recordings and video now provide the technological infrastructure that made, for instance, conversation analysis possible and today allows multi-modal observation of micro-genetic mechanisms of interaction and learning. Computer logs offer the further possibility of automatically recording unlimited amounts of high quality data for the analysis of group cognition.

For instance in our study of the case shown in Fig. 1, we used a re-play application that lets us step through exactly what was shared by everyone in the chat room. Our replayer shows the window as the participants saw it and adds across the bottom controls to slow, halt and browse the sequential unfolding of the interaction. This not only allows us to review interesting segments in arbitrarily fine detail in our group data sessions, but also allows us to make our raw data available to other researchers to evaluate our analyses. Everyone has access to the complete data that was shared in the students' original experience. There are no selective interpretations and transformations introduced by camera angles, lighting, mike locations, transcription or log format.

Of course, the analysis of group interaction necessarily involves interpretation to understand the meaning-making processes that take place. The analyst must have not only general human understanding, but also competence in the specific discourse that is taking place. To understand Aznx's utterances, an analyst must be familiar with both the "form of life" of students and the math objects they are discussing. As Wittgenstein (1953, p. 223, §IIxi) suggests, even if a lion could speak, people would not understand it. Sfard's talk about analyzing discourse from the perspective of an analyst from Mars is potentially misleading; one needs thick descriptions (Geertz 1973; Ryle 1949) that are meaning-laden, not "objective" ones (in what discourse would these be expressed?).

Sfard's discussion of the researcher's perspective (p. 278f) is right that analysis requires understanding the data from perspectives other than those of the engaged participants - for instance, to analyze interactional dynamics and individual trajectories. However, it is important to differentiate this removed, analytic perspective (that still understands the meaning making) from a behaviorist or cognitivist assumption of objectivity (that recognizes only physical observables or hypothetical mental representations). The analyst must first of all understand the discourses in order to "explore" it from an outsider's metadiscourse, and neither a lion nor an analyst from Mars is competent to do so.

Sfard defines the unit of analysis as the discourse (p. 276). The use of CSCL media for math discourses problematizes this, because the discourse is now explicitly complex and mediated. Although Sfard has engaged in classroom analyses elsewhere, in this book her examples are confined to brief dyadic interchanges or even utterances by one student. In fact, some examples are made-up sentences like linguists offer, rather than carefully transcribed empirical occurrences. Moreover, the empirical examples are generally translated from Hebrew, causing a variety of interpretive problems and lessening the ability of most readers to judge independently the meaning of what took place. Computer logs allow us to record and review complex interactions involving multiple people over extended interactions. The unit of analysis can be scaled up to include: groups larger than dyads (Fuks et al. 2006), the technological infrastructure (Jones et al. 2006), the classroom culture (Krange and Ludvigsen 2008), time stretches longer than a single session (Sarmiento and Stahl 2008). One can observe complex group cognitive processes, such as problem-solving activities - from group formation and problem framing, to negotiation 
of approach and sketching of graphical realizations, to objectification and exploration of visual signifiers, to reflection and individualization. The encompassing discourse can bring in resources from the physical environment, history, culture, social institutions, power relationships, motivational influences, collective rememberings - in short, what activity theory calls the activity structure or actor-network theory identifies as the web of agency.

While Sfard uses the language of sweeping discourses-like the discourse of mathematics from the ancient Greeks to contemporary professional mathematicians - her specific analyses tend to minimize the larger social dimension in favor of the immediate moment. This is particularly striking when she uses terms like alienation and reification to describe details of concept formation. These terms are borrowed from social theory-as constructed in the discourses of Hegel, Marx and their followers, the social thought of Lukacs, Adorno, Vygotsky, Leontiev, Engeström, Lave, Giddens, Bourdieu. Sfard describes the reification of discursive counting processes into sentences about math objects named by nouns as eliminating the human subject and presenting the resultant products as if they were pre-existing and threatening. She does this in terms that all but recite Marx's (1867/1976, pp. 163-177) description of the fetishism of commodities. However, whereas Marx grounded this process historically in the epochal development of the relations of social practice, the forces of material production and the processes of institutional reproduction, Sfard often treats mathematics as a hermetic discourse, analyzable independently of the other discourses and practices that define our world, although in her concluding chapter she emphasizes the need to go beyond this in future work.

Mathematics develops - both globally and for a child - not only through the interanimation of mini-discourses from different personal perspectives, but also from the interpenetration of macro-discourses. Math is inseparable from the world-historical rise of literacy, rationalism, capitalism, monotheism, globalization, logic, individualism, science and technology. CSCL theory must account for phenomena across the broad spectrum from interactional details contained in subtle word choices to the clashes of epochal discourses. While Sfard has indicated a powerful way of talking about much of this spectrum, she has not yet adequately located her theory within the larger undertaking. One way to approach this would be to set her theory in dialog with competing participationist theories in CSCL and the learning sciences.

\section{Continuing the discourse}

Issues of situating math discourse in social practice return us to the quandary of the metaphors of acquisition and participation. Sfard's book works out an impressive edifice of participation theory. Math can be conceptualized as a discourse in which people participate in the social construction of math objects; because of such participation they can understand and individualize elements of the discourse. In doing so, she follows a path of dialogical and discursive theory starting at least with Bakhtin, Vygotsky and Wittgenstein, and propounded by numerous contemporaries. Within the domain of math discourse, Sfard has pushed the analysis significantly further.

Her argument 10 years ago was that there is something to the metaphor of objects of math but that the ontological status of such objects was unclear and was perhaps best described by AM. In addition, she felt that multiple conflicting metaphors breed healthy dialog. But now she has shown that math objects are products of math discourse (so they now exist and make sense within PM). As for healthy dialog, there is plenty of opportunity for controversies among multiple discourses within PM itself. Thus, we can conclude that 
Sfard is justified in moving to a fully PM metaphor because this stream of thought is capable of resolving former quandaries and it contains within itself an adequate set of potentially complementary, possibly incommensurable discourses to ensure a lively and productive on-going debate. Sfard has provided us with one of the most impressive unified, homogenous theories of learning; it remains for us to situate that theory within the specific field of CSCL and within the broader scope of competing theoretical perspectives. This includes extending and applying her analysis to group cognition and to computer-mediated interaction. It also involves integration with a deeper theoretical understanding of social and cultural dimensions.

At the other end of the spectrum, one must also resolve the relationship of "thinking as communicating" with the psychological approach to individual cognition as the manipulation of private mental representations. Is it possible to formulate a cognitivist view without engaging in problematic acquisitionist metaphors of a "ghost in the machine" (Ryle 1949)? Assuming that one already understands the mechanisms of math discourse as Sfard has laid them out, how should hypothetical-deductive experimental approaches then be used to refine models of individual conceptualization and to determine statistical distributions of learning across populations? Questions like these raised by the challenge of Sfard's book are likely to provoke continuing discourse and meta-discourse in CSCL-and in $i j C S C L$ - for some time to come, resolving intransigent quandaries and building more comprehensive (deeper) scientific understandings.

\section{References}

Fuks, H., Pimentel, M., \& de Lucena, C. J. P. (2006). R-u-typing-2-me? Evolving a chat tool to increase understanding in learning activities. International Journal of Computer-Supported Collaborative Learning (ijCSCL), 1(1), 117-142.

Geertz, C. (1973). The interpretation of cultures. New York, NY: Basic Books.

Husserl, E. (1936/1989). The origin of geometry (D. Carr, Trans.). In J. Derrida (Ed.), Edmund Husserl's origin of geometry: An introduction (pp. 157-180). Lincoln, NE: University of Nebraska Press.

Jones, C., Dirckinck-Holmfeld, L., \& Lindström, B. (2006). A relational, indirect and meso level approach to design in CSCL in the next decade. International Journal of Computer-Supported Collaborative Learning (ijCSCL), 1(1), 35-56.

Krange, I., \& Ludvigsen, S. (2008). What does it mean? Students' procedural and conceptual problem solving in a CSCL environment designed within the field of science education. International Journal of Computer-Supported Collaborative Learning (ijCSCL), 3(1), 25-52.

Lockhart, P. (2008). Lockhart's lament. MAA Online, 2008 (March). Retrieved from http://www.maa.org/ devlin/devlin 03 08.html.

Marx, K. (1867/1976). Capital (B. Fowkes, trans. vol. I). New York, NY: Vintage.

Moss, J., \& Beatty, R. A. (2006). Knowledge building in mathematics: Supporting collaborative learning in pattern problems. International Journal of Computer-Supported Collaborative Learning (ijCSCL), 1(4), 441-466.

Ryle, G. (1949). The concept of mind. Chicago, IL: University of Chicago Press.

Sarmiento, J., \& Stahl, G. (2008). Extending the joint problem space: Time and sequence as essential features of knowledge building. Paper presented at the International Conference of the Learning Sciences (ICLS 2008), Utrecht, The Netherlands.

Sfard, A. (1998). On two metaphors for learning and the dangers of choosing just one. Educational Researcher, 27(2), 4-13.

Vygotsky, L. (1930/1978). Mind in society. Cambridge, MA: Harvard University Press.

Vygotsky, L. (1934/1986). Thought and language. Cambridge, MA: MIT Press.

Wittgenstein, L. (1944/1956). Remarks on the foundations of mathematics. Cambridge, MA: MIT Press.

Wittgenstein, L. (1953). Philosophical investigations. New York, NY: Macmillan. 